\title{
Rumiando realias y otros intraducibles del léxico geográfico oral del Sáhara Occidental
}

'Realias' and other no translatable expressions from the geographical oral lexicon of Western Sahara

\section{LAROSI HAIDAR}

\section{UNIVERSIDAD DE GRANADA (ESPAÑA) lhaidar@ugr.es}

Investigador, profesor y escritor saharaui. Tras el alto el fuego, se instaló en Granada, donde se licenció y doctoró en Traducción e Interpretación. Actualmente es profesor de esta misma disciplina en la Universidad de Granada y ha publicado varios trabajos relacionados con la cultura saharaui. También ha participado en varias antologías de poesía saharaui.

RESUMEN: La esencia de este trabajo son las palabras que vamos a tratar de abordar y dilucidar en el ámbito de un contexto muy concreto y limitado geográfica y culturalmente: expresiones y denominaciones pertenecientes a la lengua hassanía, variante árabe hablada en el Sáhara Occidental, referidas a la geografía y al relieve de dicho territorio. Por lo tanto, se trata de un léxico específico perteneciente a la tradición oral del pueblo saharaui y que ha ido pasando de generación en generación, acumulando, con el correr de los años, datos, matices y connotaciones que no debemos ignorar a la hora de nombrar o citar cualquier duna, pozo o acacia de cualquier rincón perdido del desierto. Son los llamados realias, palabras difíciles de traducir, por no decir intraducibles.

Palabras Clave: Traducción, Realias, Geografía, Sáhara Occidental, Cultura oral.
ABSTRACT: The essence of this work are the words that we'll try to address and clarify the scope, in a very specific and geographical and cultural limited context: expressions and denominations belonging to the Hassaniya language, Arabic variant spoken in Western Sahara, based on the geography and relief of the territory. Therefore, it is a specific lexicon belonging to the oral tradition of the Saharawi people and which has been passed from generation to generation, accumulating, over the years, data, nuances and connotations that we should not ignore when name or cite any dune, well or acacia lost in any corner of the desert. They are called realias, difficult words to translate, if not untranslatable.

KEY WORDS: Translation, Realias, Geography, Western Sahara, Oral Culture 


\section{Introducción}

Todo aquel que se ha enfrentado a la traducción de una lengua a otra, de una cultura a otra, es consciente de la existencia de palabras y expresiones reacias a ser trasladas, a ser transportadas a otra lengua mediante los rudimentos traslaticios que solemos utilizar a diario; palabras que obligan al traductor a la adopción de medidas y usos mucho más especiales, como es la transcripción sin alteraciones, la conservación de la estructura morfológica o fonética, o incluso la sustitución por unidades léxicas de muy diferente valor. Suelen ser denominaciones referentes a la vida cotidiana, a la cultura, etc., de un pueblo o territorio específico y que están ausentes en otros pueblos y lugares (Vlahofs y Florins, 1969:432)1 . "Son precisamente esas palabras las que en traductología han recibido el nombre de 'realia"' (1969: 432).

Y son, también, las palabras que vamos a tratar de abordar y dilucidar en el ámbito de un contexto muy concreto y limitado geográfica y culturalmente: expresiones y denominaciones pertenecientes a la lengua hassanía, variante árabe hablada en el Sáhara Occidental, referidas a la geografía y al relieve de dicho territorio. Por lo tanto, se trata de un léxico específico perteneciente a la tradición oral del pueblo saharaui y que ha ido pasando de generación en generación, acumulando, con el correr de los años, datos, matices y connotaciones que no debemos ignorar a la hora de nombrar o citar cualquier duna, pozo o acacia de cualquier rincón perdido del desierto.

Las palabras (y las locuciones compuestas) de la lengua popular que representan denominaciones de objetos, conceptos, fenómenos típicos de un ambiente geográfico, de una cultura, de la vida material o de las peculiaridades histórico sociales de un pueblo, una nación, un país o una tribu, y que por esa razón son portadoras de un matiz nacional, local o histórico, carecen de correspondencia precisa en otras lenguas (Vlahofs y Florins, 1969: 438).

Para la obtención de las palabras que nos servirán como muestra de denominaciones intraducibles o difíciles de traducir, nos basaremos en el primer trabajo realizado sobre geografía del Sáhara Occidental cuyo contenido, con excepción de una mínima parte, ha sido obtenido íntegramente de la tradición oral del pueblo saharaui. Se trata del libro de Buchar Ahmed que recoge la tradición oral relativa al relieve, la fauna y la flora del Sáhara Occidental y cuyo título, traducido del árabe, sería Geografía del Sáhara Occidental'. El propio autor, octogenario, es consciente de la ausencia de bibliografía sobre el tema, con lo cual justifica su apoyo en la tradición oral:

\footnotetext{
${ }^{1}$ Citado en Logos non solo parole.

${ }^{2}$ El libro ha sido publicado en 2012 en España, en lengua árabe, al no obtenerse la licencia de las autoridades marroquíes para su publicación en los Territorios Ocupados del Sáhara Occidental. En la actualidad, se está realizando su traducción al español y podríamos considerar el presente artículo como una pequeña muestra representativa del contenido de la obra.
} 
Larosi Haidar. Rumiando realias...

Este hecho hizo que en muchos casos me apoyara en los testimonios de los autóctonos del territorio quienes, evidentemente, habían caminado a lo largo de sus uads y sus colinas, habían atravesado sus montañas y llanuras, vivieron cerca de sus puntos de agua; se especializaron en el conocimiento de su fauna y su flora, comerciaron con la sal de sus salinas y lo conocen de manera exhaustiva y precisa. Sin olvidar mi propio y modesto conocimiento del mismo. (Ahmed, 2012: 9)

A fin de cuentas, lo que vamos a realizar en las siguientes líneas es manifestar explícitamente toda la carga semántica implícita en las palabras del corpus elegido, es decir, una expansión de sus connotaciones de tal manera que cualquier lector pueda concebir la referencia completa a la que aluden. Para más sencillez, evitaremos la transcripción fonética de las denominaciones hassaníes y nos contentaremos con transcribirlos en caracteres latinos tal y como sonarían en castellano, con excepción de la palatal aproximante (jota francesa) que transcribiremos como “ $\hat{y}$ ”.

\section{Denominaciones genéricas del relieve}

Entre las grandes extensiones de terreno llano y homogéneo existentes en el Sáhara Occidental, destacan las grandes llanuras, como son la de Tarfaya, que se extiende desde el río Draa hasta la región de Cabo Bojador; y la llanura de Villa Cisneros (Dajla) que va de la ciudad del mismo nombre hasta el Cabo Blanco. Además, están las numerosas llanuras discontinuas, como son la de Adraa Al-jail, la de Hassia, la de Labtteina, la de Aŷnaba y la de Lamseh. Además está la llanura de Amseh Am-mat At-tarfa que está separada de Lamseh por la elevación de Arbig Taguyás, donde fue enterrado el conocido Bilal ben Embarek.

Bilal ben Embarek ben Esiid ben Belqasem, es el ascendiente de la familia Ahl Bilal, herido de bala en la batalla de Twedarist, entre la tribu de Izarguiyín y las de Eit Bel-la, y apuñalado hasta doce veces. Milagrosamente, no murió.

Sin olvidar la llanura de Magsam Uld Amreizig, denominada así por Omar Uld Amreizig, que fue uno de los héroes de la tribu de Sal-lam y uno de sus principales líderes guerreros.

\section{Accidentes geográficos menores}

En este apartado, trataremos los nombres de gran parte de los accidentes geográficos menores, además de los regs y los ergs.

Un reg, que los autóctonos denominan aftut o salb, es una especie de llanura seca que generalmente incluye depresiones del tipo grara y agarýy, a veces, luýa y agtti, atravesada por estepas y cursos torrenciales. 
Grara: es un elemento topográfico característico del Sáhara Occidental en las zonas de regs (aftuts) consistente en una depresión cerrada de tierra limosa, cohesionada e impermeable que se cubre de vegetación tras cada periodo de lluvias. Los habitantes suelen explotarla como terreno de siembra por ser muy fértil. La planta típica de las graras es el solemne aýdari, es decir, la Rhus tripartitum Ucria.

Agary: similar a la grara, suele ser más pequeño y generalmente no contiene aýdari.

Luýa: depresión parecida a la grara, sin embargo, su tierra es permeable, no cohesionada e inválida para la siembra. Suele estar cubierta de acacias (talh) y, a veces, cuando se trata de zonas de regs (salbs) la pueblan plantas iganín, que es la denominación local de la Capparis decidua ( Forsk.) Edge.

Agtti: similar a la luýa pero de menor tamaño, se caracteriza por ser un terreno permeable.

El erg, generalmente llamado por los autóctonos adhraa, es una extensión de terreno cubierto de dunas y colinas de arena eólica proveniente originariamente de las playas marinas. En el Sáhara Occidental hay varios ergs, de los cuales algunos están formados por dunas (aghrad) y otros por una especie de médanos en tierra firme denominados en hassanía aalab.

Las dunas o aghrad suelen aparecer aglomeradas o separadas en una sucesión continua y unidireccional, siendo la media luna su forma más extendida en el Sáhara Occidental. Por el efecto del viento, se desplazan continuamente, aunque de manera tan lenta que apenas lo perciben los lugareños, con la salvedad de quienes abandonan el lugar y vuelven pasado mucho tiempo.

Entre los ergs o adhraas más conocidos podemos citar Adhraa Asátef: se extiende desde las zonas costeras de Ajneifís y Alghard Lahmar ${ }^{3}$ en el norte hasta Saguia El Hamra en el sur, que es un importante territorio de pastoreo de más de tres mil kilómetros cuadrados. En este mismo adhraa y a unos diez kilómetros al noroeste de la capital saharaui, Aaiún, está localizada la famosa duna de Agannur (Ghard Agannur). Se trata de la misma duna citada por el poeta Aziz Haidar en su célebre poema ¡Quién supiera!, aunque parece ser que, en realidad, hay dos dunas que reciben este mismo nombre, pues es lo que se entiende de los versos de Aziz:

\footnotetext{
"Mas lo que de veras me preocupa en este instante es si Agannur del Norte en su lugar está y si lo está el del Sur, famoso y grande ¿y esas sus jaimas vecinas, lo están? ¡Quién supiera!"4
}

\footnotetext{
${ }^{3}$ Literalmente significa Duna roja. "Alghard" es el singular del ya visto en forma indeterminada y plural aghrad (dunas).

${ }^{4}$ El poema completo está recogido en (ACDPM, 1995:135).
} 


\section{Elevaciones rocosas}

1. Algalb (corazón), algara (colina) y as-sattla (olla), de significado parecido y que se refieren a pequeñas elevaciones de diferentes formas y aspectos.

2. Aljashm (nariz), attaraf (saliente) y ar-rass (cabeza), son protuberancias rocosas que destacan en el extremo de una meseta o cadena de elevaciones.

3. Lagdam (talón), es un saliente situado al inicio de una meseta o elevación mayor.

4. Adh-dhalaa (costilla), elevación de forma alargada y de poca altura.

5. Az-zamla (montículo), colina alargada y de tamaño limitado.

6. At-ttoug, laadham (hueso), aganz y awzan, todos con el mismo significado de colina o elevación alargada que se prolonga cubriendo una gran distancia.

7. Alaadla, especie de colina circular muy grande.

8. Adarg, almadna y almanbá, protuberancias rocosas de tamaño limitado.

\section{Elevaciones arenosas}

1. Alghard, que es propiamente una duna.

2. Alaakla, amalgama de dunas (aghrad) entrelazadas.

3. Alaalb, especie de médano.

4. Adamán, especie de pequeña colina arenosa.

5. An-nabka, diminuta elevación arenosa mucho más pequeña que el adamán.

6. Az-zira, especie de an-nabka formada sobre una planta y de la que suelen sobresalir sus ramas. Es, por tanto, una pequeña duna anclada por efecto de la vegetación y, por ello, suele recibir el nombre de duna anclada o anchored dune.

\section{Depresiones}

En general son pequeños desniveles del terreno que dan lugar al estancamiento del agua de lluvia durante un periodo de tiempo que va de algunos días a varios meses. Además de las ya vistas más arriba, lagrara, agarý, al-luýa y agtti, están el asagh (p. isighan) que es una depresión en la que se estanca el agua y carece de vegetación. Cuando la depresión es profunda se le llama aghuŷ (p. ighuỷan) y si además es de forma relativamente alargada recibe el nombre de aýwa (p. ýuwia). Si ocupa una gran superficie es una hautta (p. hauttat); si se encuentra entre dunas es una jabtta (p. ajbatti). Recibe el nombre de sahb 
(p. sahba) cuando funciona como una especie de desaguadero poco profundo; cuando este desaguadero está prácticamente a ras del suelo se le denomina jatt (p. ajttutta). La depresión cuando es muy extensa y prolongada recibe el nombre de masrab (p. amsarab); si es estrecha, se llama at-tisram (p. at-tisramat) o también at-tiýrit (p. at-tiýrraten). Si la depresión, además de ser prolongada como el masrab, es profunda, recibe la denominación de ain (p. aaiún).

Cauces

En general, estos cauces más o menos grandes van a funcionar como ramblas por las que de vez en cuando discurre el agua de las lluvias en forma de torrentes de muy poca duración. El mayor de ellos recibe el nombre de uad (p. uadián), que sería el cauce generalmente seco de un río. Pedro Gómez Moreno usa este término en mayúsculas (UAD) y su plural (UED-DIAN), y lo define así:

río, pero que ya hemos aclarado en otra ocasión que no son tales, sino cauces por donde corre el agua acumulada o suministrada por vertientes que llegan a ellos y, como siempre, de lluvia, que hasta ahora es la mejor fuente de suministro que tienen estos territorios, pese a que se pasen algunos años sin llover. (GÓMEZ MORENO, 1959:42)

Cuando es más pequeño y muy profundo, recibe el nombre de aljawi (p. aljuwia); si es muy estrecho se le llama shalja (p. ashlaj), es decir, corte o fisura, y equivaldría a la denominación castellana de garganta. Cuando esta especie de garganta es más bien arenosa, recibe el nombre de fadra (p. afdar). Cuando el relieve es más bien un desaguadero poco profundo, se le llama almasial (p. lamsaial) o también almashrah (p. lamshárah) y cuando conducen el agua a un uad se les denomina lamkab (p. lam$k a b)$. Si en realidad no es más que una especie de falda de una elevación, recibe el nombre de mahd (p. amhad).

\section{Relieve del Sáhara Occidental con nombre propio}

Intentaremos mostrar ejemplos de algunos accidentes geográficos con nombre propio representativos del territorio saharaui.

Denominaciones con jatt (p. ajttutta $)^{5}$ :

Uad Aljatt, es uno de los más grandes afluentes de Saguia El Hamra, que nace en Assmamitt y desemboca a la altura de Itqi.

Jatt Ad-daghmús (El jatt del cactus), es el jatt que nace cerca de la tumba de Omar uld Sidahmed y desemboca en la depresión de Assbeibira (La pacientilla).

\footnotetext{
${ }^{5}$ Depresión apenas perceptible que hace de desaguadero.
} 
Jatt Attalba, nace en Dhaiat ${ }^{6}$ Am-lashrak y acaba en la depresión de Almar-ra (La amarga).

Jatt Lahwara (La ternera de camella), sale de Lamheinini-ia y acaba en Amgheidar ${ }^{7}$ Taalabutz.

Jatt Musa Laawar (Musa el tuerto), nace en Zemlat Al-amiríia para desembocar en una depresión al norte de la localidad de Lakrushi.

Lakrushi es un pequeño poblado que se construyó en los alrededores del viejo puesto fronterizo que separaba el Sáhara Español del Reino de Marruecos. De hecho, su denominación es una hassanización del castellano El Cruce, en referencia al cruce de caminos que suponía dicho puesto fronterizo, además de ser el punto de cruce legal de la frontera hispanomarroquí en la provincia española número 53. Por esos lares de la Marca de Daora, Sttalayu (el Capitán Estalayo), gran conocedor del Sáhara, su cultura y su lengua, ejercía la infalible Prueba del lobo para detectar a los posibles marroquíes que cruzaban la frontera ilegalmente haciéndose pasar por saharauis. Les ordenaba pronunciar en árabe la palabra "lobo", es decir, dib. Si la $d$ inicial era pronunciada como una oclusiva dental sonora y no como una interdental sonora no enfática, quedaba claro que el individuo en cuestión era marroquí y, por lo tanto, debía retomar el camino de vuelta a su país y documentarse como es debido.

Denominaciones con sahb (p. sahba):

Sahb Alharsha, se encuentra en Alwattia, nace en los altos de Zeini y, pasando entre Lahmediiat, desemboca en el mar.

Sahb Atwela ${ }^{8}$, nace en Zemlat Lashrak para acabar en Iwinight.

Sahb An-nil, nace en As-sakn y desemboca en Uad Aŷdari desde el norte, cerca de Ahŷeib Aghreibil. Aghreibil es el diminutivo de gharbal, que es una especie de tamiz o criba.

As-heibat Albaghlal, emanan desde el sur de Aljashbillín para desembocar igualmente en Uad Aŷdari por el norte. Significa "pequeños sahbs de los caracoles". As-heibat es el plural de as-heib que, a su vez, es el diminutivo de sahb.

As-heibat Aljeil (de los caballos), que comienzan al oeste de Ash-weishía para, a su vez, desembocar en Uad Aŷdari desde el sur. Ash-weishía es el diminutivo de shashía, gorro en forma de cubilete o fez.

\footnotetext{
${ }^{6}$ Charca, laguna.

${ }^{7}$ Charco, lagunilla.

${ }^{8}$ Atwela es el diminutivo de atila, que es la unidad de la planta atil, es decir, la Maerua crassifolia Forssk. Planta de múltiples usos, desde la cura de la diabetes y la diarrea, hasta la construcción de utensilios como cucharas y embudos. Véase Barrera Martínez (2007:20).
} 
Denominaciones con zemla (plur. zmul, dim. zmela):

Zmul An-neirán, están localizadas en las inmediaciones de Am Lagtta, en la zona de Lahmada. Significa literalmente "las colinas de los fuegos". En dicha zona de la localidad saharaui de Mahbés, se llevó a cabo el primer ataque del Ejército de Liberación Saharaui a las fuerzas de ocupación marroquíes dentro de la llamada Ofensiva Gran Magreb, en octubre de 1984. De hecho, todo el mundo en el Sáhara Occidental conoce la gran Batalla de Zmul An-neirán.

Zmélat Alghul, está situada al norte de Dhraa Medŷau y al sur de Arbett Bashafi. El ghul es un ser fantástico perteneciente a la mitología saharaui al igual que a otras mitologías. En el caso saharaui, se suele asimilar a un ente invisible que de noche ronda los cementerios o lugares donde haya tumbas. Se dice que muchas veces emite gritos horribles fácilmente audibles por los humanos, razón por la cual es el terror de los niños, pues, efectivamente, llegan a oír sus escalofriantes chillidos que van cambiando de lugar según va cambiando el ghul de posición. Sin embargo, en la mayoría de los casos, el ghul resulta ser una especie de murciélago residente habitual de la zona y, obviamente, de hábitos nocturnos.

Zmélat Agtti Adhlim, situada al norte de Zmélat Agtti At-téguatan. Adhlim es la denominación que recibe el avestruz siendo todavía joven. En el Sáhara adhlim tiene fama de ser gran bailador y así aparece en gran parte de cuentos y leyendas populares saharauis. Aunque en el pasado esta solemne ave abundaba en las estepas del Sáhara Occidental, parece que se extinguió de la región a mediados del siglo XX, pues los últimos testimonios de avistamientos e incluso de caza de avestruces datan de los años cincuenta del siglo pasado.

\section{Denominaciones con lamreýnat:}

Amreýnat es el diminutivo de amraýan que, a su vez, es el plural del término hassaní marýan, cuyo significado es olla (puchero, marmita, etc.) y tiene su origen en el árabe marýal. Obviamente, su uso en las denominaciones geográficas suele deberse al parecido que tienen ciertas colinas y elevaciones con este tipo de recipientes de cocina.

Reciben la denominación de Lamreŷnat tres cerros pétreos situados cerca de Nagŷir oriental y al norte del charco de Uen Fargán. Igualmente reciben esta denominación tres cerros arcillosos localizados en Adar, en la costa atlántica, diez kilómetros al norte de la desembocadura de Saguia El Hamra. Sin olvidar Lamhegnat, que son otros tres cerros pétreos situados al noroeste de Uashfagt. Amhegnat es el diminutivo de amhagan, plural de mahgan, es decir, embudo. 
Larosi Haidar. Rumiando realias...

Otras denominaciones serían:

Udeiat Hasi Am Lagtta (Riachuelos del Pozo de Am Lagtta). Agtta es el plural genérico de gattia (p. gattiat) ave muy común en el Sáhara Occidental. Se trata de la ganga, es decir, la Pterocles. Por otra parte, la zona de Am Lagtta, debido a su localización, ha sido escenario de muchas batallas entre las cuales tiene especial importancia la del 25/02/1987, pues fue la única vez en la que las fuerzas saharauis pudieron apoderarse de una de las armas vitales de la artillería marroquí. Se trata del obús autopropulsado de $155 \mathrm{~mm}$ de fabricación francesa.

Masial Anqab Azargui (Cauce de las brechas de Azargui). Anqab, plural de naqab, que alude a brecha, apertura, paso estrecho, etc. Azargui Ben Bel-la es el antepasado de la tribu de los Zarguiyín, muerto en combate en la batalla conocida como Batalla de las Cinco Tribus 9 .

Rass Aljanfra (Cabeza o punta de la nariz). Dicha punta se encuentra en el extremo suroeste de los altos de Zeini y, por su altura, puede ser vista desde muy lejos. En el año 1979, fue escenario de una operación emboscada en la que el Ejército de Liberación Saharaui aplastó a las fuerzas marroquíes que se encontraban en el lugar el día 24 de agosto, entre los que hubo 240 muertos y 19 fueron hechos prisioneros. Además, les fueron incautados más de 20 vehículos, 130 fusiles belgas FAL y más de 30 toneladas de munición diversa.

Uad Tiummán, que destaca por albergar varios palmerales y numerosas albercas denominadas Gregrat. La denominación se debe a la abundancia de un tipo de aves que, emitiendo su característico arrullo, acuden en bandadas para beber de las albercas. Se trata de gregar, es decir, tórtolas.

Masilat Lahbasha son masilat (cauces) que desembocan en Uad Az-zeit provenientes del Sur. En el más meridional de ellos, se encuentra la tumba del chej Sidahmad Argueibi, antepasado de la tribu de los Argueibat, convertida en un gran santuario. Cerca del mismo lugar y siempre en el mismo cauce, está ubicada la tumba del chej Sidahmad Alfeiram Alkanti.

Aaguélat Banssar (Pozo poco profundo de Banssar). Se dice que su agua es tan dulce que parece agua de la fuente Salsabil (fuente del Paraíso citada en el Corán, 76:18) y, según la leyenda popular, un profeta llegó a beber de ella. A su lado, está la tumba de Brahim bn. Abdala, antepasado de la familia de los Brahim bn. Abdala, de la fracción Suaad de la tribu de Rgueibat.

El acuífero de Abattih, de agua potable y muy abundante, próximo a un enorme palmeral considerado uno de los oasis más grandes del Sáhara Occidental. Abattih es un antiguo oasis de abundante agua potable. Por él pasaba el antiguo camino de las caravanas que surcaban el desierto. Hoy, es el paso de la carretera que une las ciudades saharauis ocupadas de Smara y Tantán.

${ }^{9}$ Véase Molina Campuzano (1954:78). 
La Julua (Soledad). Julua es el nombre que recibe una enorme cueva en la que, según la leyenda popular, el chej Sidahmed Rgueibi se dedicaba al rezo. En el manuscrito de Mohamed Salem Lehbib, titulado Ýauami almuhimat fi umur Argueibat, se dice que Sidahmed Rgueibi estuvo rezando en esta cueva durante diez años, de 1020 a 1030 de la Hégira.

Masial Udéi Arrouz (Desaguadero o cauce del Riachuelo de la Prueba). En este masial había una piedra de pedernal de forma ovalada con la que se realizaba una especie de prueba de fuerza entre los hombres. La prueba consistía en levantar la piedra hasta donde se podía. Algunos la levantaban hasta las rodillas; unos, hasta el ombligo; otros lograban incluso lanzarla hacia atrás por encima de la cabeza; mientras que algunos únicamente podían moverla de sitio haciéndola rodar. Donde mana el masial, hay una señal histórica dejada por los cascos de un caballo al saltar. Se trataría del aguerrido Mohamed Laffairas, que al verse sorprendido por el enemigo su caballo dio un salto de 30 codos, de tal manera que tuvo tiempo de defenderse disparando y, finalmente, salvarse. Esto sucedió en 1850.

Udéi Amma Fatma (Riachuelo de Amma Fatma), quien fue una mujer devota de la tribu saharaui de Maŷatt. Su tumba, convertida en santuario, se encuentra en la ribera norte del río, al igual que muchas otras tumbas de Izarguiyín y Mayaatt, como es el caso del conocido Alfeidal uald Brahim uald Ali uald Moulud. En la ribera sur, pegadas al camino, están ubicadas muchas tumbas de fallecidos izarguiyín, entre ellos Taleb Ahmed ben Brahim ben Asaid que es el antepasado de la subfracción de los Ahl Taleb Ahmed y eminente sabio religioso.

Masial Bumghara: Bumghara es, literalmente, "el de la gruta”. Se trata de una gran cueva situada en la confluencia de Masilat Lagüettrat con Uad Zahar cuya superficie alcanza la hectárea y tiene una altura de cerca de 30 metros. Por debajo de ella, corre agua salada que forma albercas o bolsas de agua, y a su lado hay un pozo algo menos salado del que bebe el ganado.

Camino de Lamŷefla (La Ahuyentadora), es un camino muy estrecho y de difícil paso al que los camellos evitan y se asustan al acercarse a él, se ahuyentan.

Garat Alhas (Cerro del ruido): según parece, por sus inmediaciones se solían oír sonidos parecidos a los que se perciben en las afamadas colinas de Laŷuad y que, sin embargo, desaparecieron tras ser enterrado en el lugar Baba uald Dada, de la fracción Boihat de los Erguibat.

Aljanga (Estrangulamiento), estrechamiento del terreno. El lugar, donde hay un pozo de agua potable, fue usado por las autoridades coloniales españolas como puesto de vigilancia. Fue el objetivo de la primera operación armada llevada a cabo por el Frente Polisario, el día 20 de mayo de 1973.

Hauza: la construcción de Hauza es una especie de torre de vigilancia de piedra, de dos pisos, localizada sobre una colina y atribuida al pueblo de los Bafour al que se le atribuyen muchas alquerías construidas de piedra. Los Bafour son los habitantes originales de la región, ancestros de los Soninké. 
Resistieron durante siglos la invasión, primero bereber y luego árabe. Ya en el siglo XVII, la Guerra de Balfour o de los Treinta Años Mauritana (1644-1674), habría sido un último esfuerzo infructuoso de resistencia ante los invasores yemenitas de las tribus de Banu Hassán.

Gléib Alfartuna (Montículo de la fortuna): gléib es el diminutivo de galb (corazón, montículo). Cerca de este montículo, tuvo lugar la legendaria batalla de Gléib Alfartuna o, como algunos la llaman, Gléib Ajshash, el 5 marzo de 1913 entre los guerreros saharauis y las fuerzas de incursión francesas. En la batalla, murieron muchos franceses, entre ellos el comandante Gérard, ayudante de campo del comandante de la fuerza, Muriel, y fueron hechos prisioneros cuarenta combatientes mauritanos alistados en el Ejército colonial francés.

Udéi Attarshán (Riachuelo de los Sordos): Attarshán es una tribu árabe mauritana. Un grupo de hombres pertenecientes a esta tribu entraron en combate con otro grupo perteneciente a la familia Ahl Brahim Ben Abdala, de la fracción As-suaad de Erguibat, en este mismo uad.

Agninat Andur: Andur es Mohamed uald Brahim uald Mohamed Idir uald Ahmad uald Amhammad uald Azargui, el abuelo antepasado de los Ahl Andur, hombre conocido por su dedicación a la agricultura y a la transformación del terreno para hacerlo cultivable.

Sabjat Am As-sanssal: recibe este nombre una sabja en al que abunda el as-sanssal. Gómez Moreno (1959:42) la define así: "SEBJA, en plural ESBAJ, es una depresión cuyo fondo, siempre salino, está normalmente por debajo del nivel del mar”. As-sanssal es la arcilla. Ésta, al ser pura, presenta un color blanquecino y suele utilizarse en las escuelas coránicas para limpiar y blanquear las tablillas de madera o aluah (sing. lauh) en las que los alumnos escriben las suras que van a memorizar.

Aadheim Am Aŷlud: Aadheim es el dimutivo de aadham, hueso, que en este contexto, como ya se dijo, hace alusión a una colina o elevación alargada que se prolonga cubriendo una gran distancia. $\mathrm{Am}$ aylud, literalmente "la madre de las pieles", lo que se debe a su uso en el curtido de pieles, es otra denominación de alghalga, es decir, la Pergularia tomentosa (véase Barrera Martínez, p. 61). Otra acepción del término am aýlud es la referida a la afección "de la piel” cuyo principal síntoma es una inflamación purulenta tipo forúnculo.

Graiar Albal-larỳiyat: Graiar es el plural de grara, y albal-larýiyat vendría a significar "las relativas a, las de, bal-laray". Bal-laraý es el nombre que recibe la cigüeña y el término provendría del griego pelargus. De esta manera, la denominación de las graras sería algo así como "Las Graras Cigüeñeras", lo que podría ser señal de que por dichas graras pasaba la trayectoria migratoria anual de las cigüeñas.

Grárat Ahl Brehmat (La grara de los Brehmat): Ahl Brehmat son una familia de la tribu ansarí de los Aulad Tidrarín, familia que arregló y acondicionó dicha grara para ser cultivable. 
Jangat Farrah (Estrechamiento de Farrah): Farrah es el nombre con que es conocido el devoto ualí Sidi Hsun Brahim Alaatabi perteneciente a la tribu de Yaaza Uiahda. Su tumba, convertida en un gran santuario, se encuentra en el lugar y se ha construido sobre ella una cúpula que todavía sigue en pie. Algo más al norte de esta janga se encuentra Jangat Alkatba (Estrechamiento de la Escritura), en la que sus rocas están cubiertas de antiguas inscripciones. Cuando llueve y las rocas se limpian por efecto del agua, las escrituras son claramente visibles.

Lahmada (La Hamada) es una extensa región que se extiende desde la zona de Aidar hasta la de Zammur, lejos del mar, lo que la hace de un calor intenso y de aire seco. Su suelo es muy permeable, por lo que escasean las aguas subterráneas a poca profundidad. Por esta razón, es conocida la maldición de ¡Que Dios te dé la sed de Lahmada! Igualmente se dice que su nombre ahmada, sin artículo, proviene de ¡ah!, que es la interjección saharaui para expresar dolor; y de ¡ma dha!, ¡cuánto!, es decir, que vendría a ser jAy, cuánto [calor, sed...]!

Quizá esta interpretación del nombre, la más conocida y difundida, no sea más que una original ocurrencia de algún nativo, pues parece ser que el término hamada, en las comunidades árabes, hace referencia a un desierto árido y sin vegetación donde sólo hay piedras. De hecho, en la terminología científica árabe utilizada en textos actuales sobre geología, es un tipo de desierto que a veces se confunde con el reg, pues la principal diferencia es la base rocosa y elevada que caracteriza a la hamada. Ejemplos de desiertos hamada los hay en varios lugares: en Libia, están la Hamada Al Hamrah y la Hamada Zeguer; en Mali, la Hamada El Harich; en Níger, la Hamada Mangeni; en Argelia, la Hamada del Draa, la Hamada de la Douara, la Hamada del Guir y la Hamada de Tinrhert; y en Jordania el desierto Hamada. Cabe destacar que el 70 \% del Gran desierto del Sáhara es del tipo hamada, siendo la mayor del mundo la citada Hamada del Draa, en el noroeste del desierto del Sáhara, ubicada entre Marruecos, Argelia y el Sáhara Occidental, y de la que formaría parte nuestra Lahmada.

\section{Las estaciones y el agua}

En lo que se refiere a los cambios estacionales, la sabiduría popular ha dejado bien definido todo lo referente a las fechas aproximadas de comienzo y fin de cada estación. En este sentido, el día 16 de marzo sería la fecha del primer equinoccio; el 27 de abril, la entrada del nisán o temporada de lluvias ${ }^{10}$; el 17 de mayo, el comienzo del verano; el 12 de julio sería el comienzo de las denominadas asmáyam ${ }^{I I}$, que son

\footnotetext{
${ }^{10}$ El periodo de lluvias nominal varía según los lugares y las tradiciones. Por ejemplo, más al sur, en ciertas partes de la vecina Mauritania, se habla de un nisán que comprende siete días de marzo (los últimos) y siete días de abril (los primeros).

${ }^{11}$ Esta es la denominación que reciben en árabe los vientos muy calientes.
} 
cuarenta días de calor extremo. El 17 de agosto es cuando empieza el otoño, siendo el 16 de septiembre el día del segundo equinoccio; el 17 de octubre es el comienzo de la temporada de siembra y el 15 de noviembre, la entrada del invierno. El 12 de diciembre sería el comienzo de las al-yali (las noches), que son cuarenta noches de frío extremo en el que se congela el agua que está a la intemperie.

Respecto a las precipitaciones que tienen lugar en el Sáhara Occidental, estas van a ser líquidas, es decir, lluvias, salvo raras veces en las que sí se precipita el agua en estado sólido, generalmente, en forma de granizo. De hecho, la denominación de granizo en hassanía es tabú, se evita su pronunciación, pues podría ser causa de una granizada con todo lo que esto supone. Imagínense en pleno desierto, donde no haya árboles ni rocas donde guarecerse y que, de repente, se pone a granizar bolas de hielo del tamaño de una pelota de tenis. Sería una muerte segura, de allí que se evite pronunciar la fatídica palabra que hace alusión al granizo en hassanía, es decir, at-tabrur.

La denominación de lluvia en hassanía es ashab, y proviene del étimo árabe referido a nube. Según la fuerza con la que cae, la lluvia puede ser rash, una llovizna muy ligera; ttash, lluvia que sigue siendo débil; ttal, una lluvia que ya rocía el terreno; naddh, lluvia que humedece y riega; $h a t t l$, lluvia recia, que cae en abundancia; y finalmente, $b a l$, fuerte lluvia que empapa y moja.

Es bien sabido que, en ciertos lugares, el agua se concentra a pocos metros del suelo gracias a la acción de algún sustrato de arcilla o material de características similares que apenas permite la filtración del agua hacia más abajo. Evidentemente, las tribus lugareñas estaban al tanto de este extremo, con lo que a lo largo del tiempo han podido descubrir varias bolsas de agua que eran verdaderos lagos subterráneos, así como manantiales y grutas que ningún mapa conocido hoy en día recoge. En lo que respecta a los tipos de puntos de agua existentes en el Sáhara Occidental, podemos distinguir diez tipos según la clasificación local: albir, alhasi, alaagla, tilamsi, algalta, alaein, albaýbaýa, ad-dhaya, almaghdar y alaushal. Estos últimos pueden ser, a su vez, agalmim, ashguig, agaýgal y tauartta.

Albir: es un pozo que sobrepasa los 15 codos de profundidad. En Gómez Moreno (1959:41) aparece como "BIR, que en plural es ABIAR, es un pozo muy profundo, cosa que interesa saber por si, al tener que hacer uso de él, contamos con los medios necesarios para llegar a su fondo". El codo fue una unidad de longitud muy utilizada en muchas culturas y, según el lugar, tenía un valor u otro, el cual podía ser de unos 38 centímetros, como el codo aragonés, hasta de unos 84 centímetros como ocurría con el codo mayor de los moriscos. En el Sáhara Occidental, al igual que los territorios vecinos, dos codos eran considerados como un metro.

Alhasi: es un pozo de menos de 15 codos de profundidad. Para Gómez Moreno (1959:42) "HASSI, en plural HASSIAN, es un pozo corriente y normal". 
Alaagla: es el pozo que tiene menos de 4 codos de profundidad. Gómez Moreno (1959:42) la describe como "AGLA -AGELI-, pozo a flor de tierra generalmente, especie de charco de agua también recogida de lluvias"(sic.).

Tilamsi: todo punto de agua que no sobrepasa la profundidad de un codo. Así la define Gómez Moreno (1959:43): “TILMSI -TILMSON-, es un agla menos profundo. Son pozos que están a flor de tierra en barrancos por los que no corre el agua, pero que está inmediata a la superficie”(sic.).

Algalta: es una alberca natural de agua permanente o temporal, dulce o salada. Gómez Moreno (1959:42) dice que "GUELTA, en plural AGLAT o LEGLAT, es una presa natural formada por el mismo terreno y que se llena de agua cuando llueve".

Alaein: manantial de agua corriente superficial, dulce y potable. Para Gómez Moreno (1959:42), “AIN -AAIUN-, fuente; ojos por los que naturalmente aparece el agua. De ahí su nombre de la capital de la provincia, asentada sobre el acantilado de la Saguia el Hamra, en donde se ven varios manantiales".

Albaýbaýa: manantial de agua corriente superficial y salobre.

Ad-dhaya: es una balsa que se llena con el agua de lluvia, conservándola durante un periodo que va de un mes a más de un año, lo que dependerá de la intensidad de las lluvias, de la profundidad de la balsa y su superficie, de la acción del viento, así como de la solidez y cohesión del terreno. Gómez Moreno (1959:42) la define como "DAIA -DI-, charco amplio que se forma en alguna ondulación o pequeña depresión. Es igualmente de lluvia y dada su amplia superficie de evaporación, se seca pronto”.

Almaghdar: es una depresión profunda en el lecho del uad o del masial que, con la lluvia, se llena de las aguas corrientes por el cauce y, una vez detenida la lluvia y el correr de las aguas, se mantiene llena como una dhaya durante un periodo de tiempo. En Gómez Moreno (1959:43) aparece como "MAGDER, charco que queda embalsado en los recodos de los ríos después de haber corrido éstos por efecto de las lluvias".

Alaushal: son un tipo de hoyos y brechas naturales localizados en un terreno rocoso compacto que, tras la lluvia, se llenan de agua que conservan durante un largo periodo de tiempo. Pueden ser de cuatro tipos: agalmim, ashguig, agaýgal y tauartta. Mohamad uald Abdalahi uald Albujari, en su manuscrito sobre la vida de la tribu de los Ahl Barikal-la, los define como una especie de hoyos naturales localizados generalmente en superficies de roca maciza y que pueden ser de tres tipos. El ashguig (p. 
ishaggan $)^{12}$, como un corte en una roca; tauartta $^{13}$, que es como un corte entre dos rocas, y arshán, que es una especie de ashguig muy profundo parecido a un pozo pequeño.

\section{Pozos y puntos de agua}

Hasi Ad-daura (Daora): es un pozo antiguo y muy importante localizado en la parte oriental de la cubeta de Daora. Fue construido por la tribu de Izarguiyín al igual que la histórica alcazaba que hay a su lado y que estuvo levantada hasta 1945, año en el que fue arrasada por las inundaciones. En aquel entonces, la cubeta de Daora se convirtió en una especie de laguna que conservó el agua durante tres años seguidos. En las proximidades del pozo y la alcazaba, tuvieron lugar en el pasado muchas batallas entre esta tribu y fuerzas agresoras provenientes de otros lugares, siendo la más conocida la habida contra las fuerzas atacantes del sultán marroquí guiadas por el alcaide Dahmán uald Beiruk, posiblemente en el año $1884^{14}$.

Tras la firma de un acuerdo entre el británico Donald Mackenzie y el líder saharaui de la tribu de Eit Musauaali, Mohamed uald Beiruk, para que el primero construya un punto de intercambio comercial en la costa de Cabo Juby (Tarfaya), el sultán marroquí Mulay Hassan, temeroso de perder el control de las caravanas que cruzan el desierto, envía al año siguiente (1880) una expedición para controlar la zona. Sin embargo, no causa ningún daño, por lo que en 1882 envía una segunda expedición de más de 20.000 hombres, que resultó en un segundo fracaso, pues murieron 6000 hombres únicamente de sed. Ya en 1888, agentes marroquíes asesinan al gerente de la factoría de Mackenzie y tienen lugar choques esporádicos entre los Izarguiyín, tribu aliada de Mackenzie, y las fuerzas del sultán marroquí comandadas por Dahmán uald Beiruk, hermano del anterior y que fue influenciado por los enviados del sultán para rebelarse.

Hasi Alayafa (Pozo de Alayafa): Alayafa fue una devota mujer de la tribu de Aulad Tidrarín de la que se decía tener la capacidad de predecir el futuro. En árabe, el término aliyafa designa la acción de agorar mediante la observación del vuelo de las aves, siendo alayafa (masc. alayaf) el nombre que recibe la mujer agorera según este método.

Es un antiguo pozo de abundante agua potable y de una profundidad de 3 metros. Se encuentra ubicado en el lecho de Uad Saguia, muy cerca de su desembocadura en el mar.

\footnotetext{
${ }^{12}$ Gómez Moreno (1959:42) ofrece la siguiente definición: “ACHGUIG, en plural ICHEGUEN, es una oquedad que absorbe el agua de lluvia y que se extrae al hacer falta y si se llega a tiempo, pues éstos suelen ser más pequeños”.

${ }^{13}$ Así la define Gómez Moreno (1959:43): “TAUERTA -POZO-, en piedra, especie de covacha, de poca profundidad, al que se puede llegar con facilidad".

${ }^{14}$ Véase García Figueras (1941) y Ruiz Miguel (1995).
} 
Hasi At-tartar (Pozo del Parloteador): At-tartar procede del árabe tartara, que significa “parlotear”. En hassanía, y partiendo de esa acepción original de parlotear, suele hacer referencia al ruido (parloteo) realizado por un motor de combustión, de allí que una de las denominaciones de la motocicleta sea at-tertar, diminutivo de at-tartar, "el parloteador".

Bir Ganduz (Pozo de Ganduz): parece ser que Ganduz, familia bereber convertida con el tiempo en tribu, vivía en el noreste de Argelia. Con las invasiones otomana, primero, y francesa después, se adentraron en el desierto donde se hicieron fuertes. Posiblemente, durante esta expansión, algunos miembros de la misma se asentaron en el Sáhara Occidental y construyeron dicho pozo. No hay indicio alguno de que exista hoy en día algún miembro de esta tribu en el Sáhara Occidental. El término tiene dos acepciones en bereber: ternero y estudiante, sin embargo, en hassanía la acepción más conocida es la que hace referencia a "trozo de carne con hueso obtenido de las extremidades del animal".

Aghuenit: se trata de un pozo antiguo localizado en el extremo sureste de la frontera saharaui mauritana, precisamente, al sur del montículo Galb Adghid. Al norte del pozo y a unos 5 kilómetros, se encuentra un aeródromo que data de la época española. En palabras de Hamad (1993:218), fue en las inmediaciones del pozo cuando, en 1294 H. (1876 d.C.), murió a manos de la tribu de Aulad Ghailán el aguerrido Brahim Aljalil, de la tribu de Aulad Dlem. Igualmente, en el año 1932 y cerca del pozo, las tropas invasoras francesas apresaron al combatiente Mohamed Rashid uald Omar uald Andur. El suceso tuvo lugar tras la famosa batalla de Tuŷunín. Se trata del ataque al grupo nómada francés de la región mauritana de Attar, perpetrado en Tuŷunín (en francés, Toujounine) el 7 de septiembre de 1931 por un ghazzi saharaui de Thalat y Suaad (Erguibat) con un chej de Ahl Dieh Almojttar (al mando del ghazzi estaban Mohamad Almamún bn. Mohamad Fadel y Ahmad bn. Hamadi). Las represalias por parte de las fuerzas coloniales francesas no se harían esperar, sobre todo, durante el año 1932.

Aaglat Aghzala (Pozo de Gacela): Aghzala (Gacela) es el nombre del caballo del devoto ualí Aabeid uald Salam, de la tribu saharaui de Yaggut, cuya tumba se encuentra en el lugar. Se dice que Aghzala fue quien escarbó en el suelo e hizo brotar el agua.

Tilamzún: en este lugar están las ruinas de antiguas construcciones, así como las de una mezquita, de las que se dice que han sido obra de la tribu árabe Yaggut. Igualmente, está la cúpula del devoto ualí Sidi Daud de Yaaza Uiahda. También, se dice que fue Hmad uald Bakkar, de Yaggut, quien excavó y encontró agua. Al sur de Tilamzún, se encuentra una roca erguida de talla mayor que la de un hombre denominada Roca de la Cristiana. Se cuenta que tuvo lugar en la zona una batalla entre saharauis y portugueses. Entonces, la esposa del comandante de las fuerzas portuguesas se puso encima de la roca y empezó a gritar para animar a las tropas hasta que fue herida y murió sobre la misma roca. Su sangre corrió sobre la roca y acabó convirtiéndose en una brillante mancha oscura. Se dice que la persona que la mató fue Alhasan uald Alal, de los Eit Hammu Hmad uald Bakkar, de Yaggut. 
Tilamzún fue en el pasado un manantial de agua corriente y, actualmente, es una balsa de agua medianamente salada y con varios pozos a su alrededor. Es muy frecuentada y su agua es abundante, estando localizada en el camino que une la ciudad de Tantán y la alquería de Lamsayad.

Hasi Aghmuga (Pozo de Aghmuga): Aghmuga es el nombre de un hombre de la tribu de Lahmanat que encabezaba un destacamento de combatientes de su tribu en una batalla contra hombres de la tribu de Yaggut. Entre los caídos ese día en combate, cerca del pozo, se encontraba el jefe del destacamento Aghmuga. Desde entonces, el pozo recibió su nombre.

Bir Aljaulat (Pozo de Aljaulat): se trata de Aljaulat uald Ambarak de Eit Iburk, de los Yaggut, que es el antepasado de la subfracción de Ahl Aljaulat.

Ab-buer Gáf-fadh: Ab-buer es el diminutivo de bir, y gáf-fadh es el imperativo del verbo "remangar [los pantalones]". Así, la denominación del bir vendría a significar "El Pocito Remángate los Pantalones", lo que sería una referencia a lo trabajoso que resulta sacar agua del mismo.

\section{Conclusiones}

Evidentemente, y según hemos visto a lo largo de las páginas anteriores, los referentes presentados como denominaciones de diferentes elementos geográficos de la cultura saharaui, no son más que la punta de un inmenso iceberg de significaciones y connotaciones latentes que el usuario de la lengua hassanía sabrá activar y desactivar según las situaciones, los contextos, los interlocutores, etc. En este mismo sentido, el traductor de estas palabras, de estos realias, deberá enfrentarse a esa misma labor de usuario de la lengua y explicitar de manera clara e ilustrativa todos aquellos aspectos que el contexto aconseje activar y hacer llegar al nuevo lector. No es tarea fácil, máxime cuando se trata de un ámbito lingüístico cultural apenas difundido y especialmente marginado. En trabajos anteriores y siempre con el ánimo de contribuir a una mejora del conocimiento, hemos intentado expresar nuestros puntos de vista, nuestras consideraciones y reservas referentes a producciones que de alguna manera tocaban el ámbito cultura saharaui (véase Haidar 2012; 2014; 2016). Un caso singular es el último de ellos, pues la total ausencia de información referente a las condiciones de producción del texto tratado así como de su traducción, apenas dejaba margen para llegar a conclusiones exhaustivamente fundamentadas. En dicho artículo describía someramente y planteaba cuestiones sobre la traducción del manual Sáhara: libro de lectura bilingüe para EGB del autor gaditano Fernando Luis Álvarez Crespo. Especialmente, destacaba la anomalía de traducir Bir Nzaran (denominación que hemos visto más arriba) como Pozo del Cristiano, lo cual me pareció extraño y poco profesional. Sin embargo, poco tiempo después de la publicación de mi artículo, el destino quiso que cayera en mis manos información pormenorizada sobre el libro y las peripecias que rodearon su publicación. 
Destacaré dos hechos relevantes para nuestro caso. Primero, el manual fue idea y trabajo individual de una sola persona que, en ningún momento, contó con un equipo de revisores o correctores, y que por amor a sus alumnos saharauis quería ofrecerles conocimiento de su propia tierra y cultura en su propia lengua, además de la española. Todo esto, en el mismo año en el que ya se olía en el ambiente que España estaba a punto de poner los pies en polvorosa del Sáhara Occidental. Segundo, y esto es lo que explicaría mi extrañeza ante la traducción ofrecida en el libro, es que los trabajos de traducción se llevaron a cabo, en el periodo de vacaciones, por un equipo de dos estudiantes árabes que ni siquiera eran saharauis. Habida cuenta de esta información, y otras que no ha lugar su cita aquí, sólo me queda felicitar al autor por su obra así como lo hizo el Sr. Secretario General del Gobierno de Sáhara concediéndole, el 24 de marzo de 1975, un premio por tan elogiable tarea:
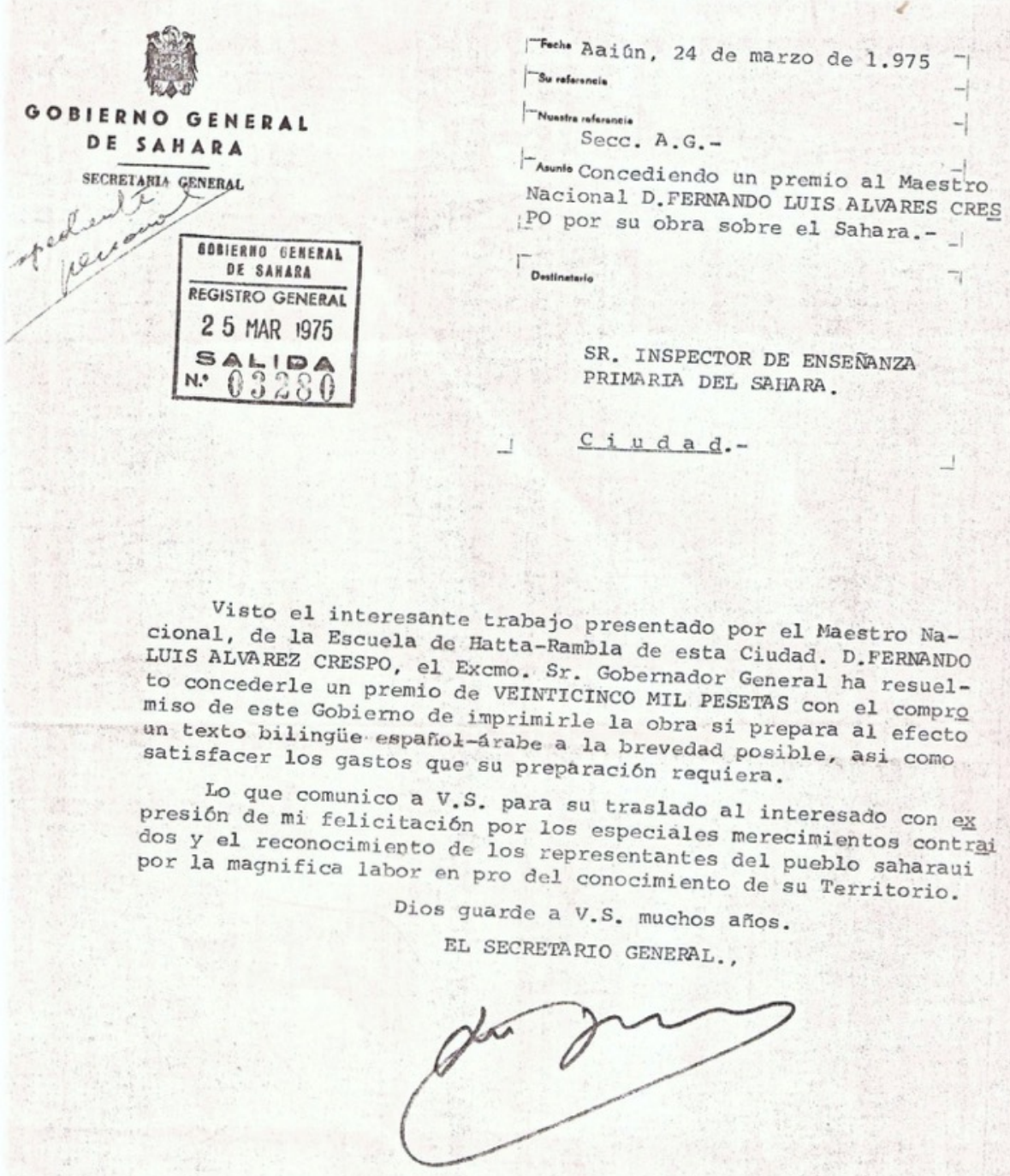
Lástima que gran parte de los diez mil ejemplares de la primera y única edición del libro fueran secuestrados por los primeros contingentes de las fuerzas de ocupación marroquíes que entraron en la capital del Sáhara Occidental.

Este es un caso claro de la punta del iceberg que apenas nos deja sospechar la inmensidad del mismo sumergida bajo la oscuridad y el desconocimiento. Lo mismo ocurre con las palabras y denominaciones que hemos tratado aquí, los realias, diminutas puntas de gigantescos icebergs de significaciones y referencias que deberemos tratar en todo momento con rigor y sutileza si no queremos acabar como un rocambolesco Titanic encallado en las arenas del desierto. 


\section{Bibliografía}

Asociación para la Cooperación y el Desarrollo con los Países del Magreb (ACDPM) (1995). Sáhara: 20 años bastan. Gijón: La Industria.

Ahmed Haidar, Buchar. (2012). Geografía del Sáhara Occidental (en lengua árabe). Madrid: Bubok.

Barrera Martínez, Ildefonso et al (2007). Sáhara Occidental: plantas y usos. Madrid: A.S. Comunicación.

García Figueras, Tomás (1941). Santa Cruz de Mar Pequeña-Ifni-Sahara (la acción de España en la costa occidental de África). Madrid: Fe.

Gómez Moreno, Pedro (1959). Pozos del Sáhara. Madrid: Consejo Superior de Investigaciones Científicas.

Haidar, Larosi. "Anomalías traductológicas en Proverbios saharauis". Tebeto: Anuario del Archivo Histórico Insular de Fuerteventura 20 (2012): 373-386.

Haidar, Larosi. (2014). "La esclavitud en el documental STOLEN: ¿esclavitud traducida o traducción esclavizada?”. MARTÍN CASARES, Aurelia (eda.). Esclavitudes hispánicas (siglos XV al XXI: horizontes socioculturales). Granada: Universidad de Granada: 283-298.

Haidar, Larosi. “La traducción en el manual Sáhara: libro de lectura bilingüe para EGB”. Entreculturas 7-8 (2016): 21-34.

Hamad, Almujttar (1993). Hayat Muritania: "alyughrafiya" (La vida de Mauritania: Geografía). Rabat: Instituto de Estudios Africanos.

Molina Campuzano, Miguel (1954). Contribución al estudio del Censo del Sáhara. Madrid: CSIC Instituto de Estudios Africanos.

Ruiz Miguel, Carlos (1995). El Sáhara Occidental y España: historia, política y derecho. Análisis crítico de la política exterior española. Madrid: Dykinson.

Vlahov, S. y Florin, S. “Neperovodimoe v perevode. Realii”. Masterstvo perevoda 6 (1969): 432-456. 